\title{
Target regimen profiles for treatment of tuberculosis: a WHO document
}

\author{
Christian Lienhardt ${ }^{1}$, Payam Nahid ${ }^{2}$, Michael L. Rich ${ }^{3,4,5}$, Cathy Bansbach ${ }^{6}$, \\ Emily A. Kendall ${ }^{7}$, Gavin Churchyard $^{8,9}$, Lice González-Angulo ${ }^{1}$, \\ Lia D’Ambrosio ${ }^{10}$, Giovanni Battista Migliori (10 ${ }^{10}$ and Mario Raviglione ${ }^{1}$
}

Affiliations: ${ }^{1}$ Global TB Programme (GTB), World Health Organization, Geneva, Switzerland. ${ }^{2}$ Division of Pulmonary and Critical Care Medicine, University of California, San Francisco, San Francisco General Hospital, San Francisco, CA, USA. ${ }^{3}$ Division of Global Health Equity, Brigham and Women's Hospital, Boston, MA, USA. ${ }^{4}$ Harvard Medical School, Boston, MA, USA. ${ }^{5}$ Partners In Health, Boston, MA, USA. ${ }^{6}$ Global Health Program, Bill \& Melinda Gates Foundation, Seattle, WA, USA. ${ }^{7}$ Division of Infectious Diseases, Johns Hopkins University School of Medicine, Baltimore, MD, USA. ${ }^{8}$ Aurum Institute, Johannesburg, South Africa. ${ }^{9}$ School of Public Health, University of Witwatersrand, Johannesburg, South Africa. ${ }^{10}$ World Health Organization Collaborating Centre for Tuberculosis and Lung Diseases, Maugeri Care and Research Institute, IRCCS, Tradate, Italy.

Correspondence: Christian Lienhardt, GTB, World Health Organization, 20, Avenue Appia, CH-1211 Geneva 2. Switzerland. E-mail: lienhardtcawho.int

@ERSpublications

Newly launched WHO target regimen profiles for new anti-TB regimens: process and results of this initiative http://ow.ly/TOUO307po08

Cite this article as: Lienhardt C, Nahid P, Rich ML, et al. Target regimen profiles for treatment of tuberculosis: a WHO document. Eur Respir J 2017; 49: 1602352 [https://doi.org/10.1183/13993003.02352-2016].

Treatment of tuberculosis (TB) relies on a combination of bactericidal and sterilising drugs administered for an adequate duration of time to ensure synergy of action in order to achieve definitive cure and prevent the selection of drug-resistant mutants [1]. Current treatment regimens are, however, not ideal, due to long duration and some toxicity. Particularly unsatisfactory are regimens recommended for the treatment of multidrug-resistant (MDR) (a form of tuberculosis with strains resistant to at least rifampicin and isoniazid) or extensively drug-resistant (XDR)-TB that have lower efficacy, significant toxicity, longer duration and high costs [2-5]. New TB drugs and regimens are needed to improve cure rates, lessen toxicity, and shorten the treatment of both drug-susceptible (DS) and drug-resistant TB (currently at least 6 and 9-20 months, respectively) [6, 7]. Two TB drugs (bedaquiline and delamanid) [8, 9] have become available and are recommended by the World Health Organization (WHO) for the treatment of MDR-TB under certain conditions $[10,11]$. However, these drugs have so far been tested for efficacy as add-ons to conventional WHO-recommended treatment for MDR-TB only, and their optimal use in combinations that could lead to increased treatment efficacy while improving safety and reducing toxicity still remains to be established [12-14]. Other novel compounds, as well as re-purposed drugs, are currently in clinical trials, either as part of novel treatment regimens or in addition to the current standard of care [15].

The development of TB drugs is lengthy and costly: if new drugs are added to or substituted within existing regimens one at a time, it would take 15-20 years to develop a new regimen of three to four new drugs to treat TB $[6,7]$. Developing a novel regimen without going through intermediary steps to obtain individual drug approvals separately and only then beginning to test novel combinations would substantially reduce the duration of the whole regimen development. It will also reduce the expenditures needed to make significant progress in the field $[16,17]$. Combination regimens including one or more promising new or re-purposed drugs should be tested early in the clinical development process to identify

Received: Nov 302016 | Accepted: Dec 012016

Conflict of interest: Disclosures can be found alongside this article at erj.ersjournals.com

The content of this work is copyright of the authors or their employers. Design and branding are copyright @ERS 2017. 
optimal combination regimens for the treatment of DS and drug-resistant TB to be tested in phase II and III trials.

Development of shorter and simpler regimens combining new and existing drugs requires detailed information on their respective activity, safety and toxicity [18-20], including potential drug-drug interactions, propensity for development of drug resistance while on therapy [21-24] and clinical use in specific patient populations (such as persons with HIV/AIDS, pregnant women and children [4]). Given these complexities, target product profiles (TPPs) are usually composed as a guide to identifying desired product attributes and characteristics to be considered during the development process [25].

The aim of this editorial is to discuss the concept and role of TPPs for new anti-TB regimens, referred hereafter as "target regimen profiles" (TRPs), and describe the outcomes of the recent process led by WHO to develop these profiles [26].

\section{Objective and role of TRPs for TB treatment}

Considering the need for safer, simpler, more efficacious and accessible treatment regimens for all forms of $\mathrm{TB}$, TRPs for TB treatment have been developed in a synergistic effort by the WHO Global TB Programme and the WHO Task Force on New TB Drug Policy Development, with the contribution of a large range of experts and stakeholders.

The novelty of this approach is to have the goal of the treatment regimen in mind very early in the process of drug development, and relies on the fact that $\mathrm{TB}$ drug research and development should move rapidly towards developing and testing TB regimens rather than individual drugs. On this basis, TRPs for TB treatment have been developed to describe the targets and specifications that developers should meet for appropriate performance and adequate operational characteristics of new $\mathrm{TB}$ treatment regimens, considering the needs of end-users. At a minimum, the TRPs specify the clinical indication of the regimen(s), the goal to be met, the measure of efficacy, the main safety aspects, the target population that will receive the treatment and the intended end-users. In addition, they should outline the most important performance and operational characteristics, with the term "minimal" used to refer to the lowest acceptable output for a characteristic and "optimal" used to refer to the ideal target for a characteristic. The optimal and minimal characteristics define a range: it is therefore expected that new TB treatment regimens meet at least all of the required minimal characteristics and, preferably, as many of the optimal characteristics as possible.

The target audience includes the pharmaceutical industry, academia, research institutions, product development partnerships, nongovernmental organisations, and potential investors and donors [26].

\section{Methods to develop the TRPs}

The initial profiles were drafted on the basis of outcomes of expert group meetings, an initial stakeholder survey, mathematical modelling, and interviews of a wide range of experts and stakeholders.

First, the candidate regimen will consist of a minimum combination of drugs targeting all possible populations of bacilli in the patient (i.e. including those proliferating in local acidic conditions or in states of brief sporadic metabolism or replication) and having a clear sterilising effect (so as to ensure nonrelapsing cure within a few months after starting the treatment). In defining the potential TRPs, the Xpert MTB/RIF assay (Cepheid, Sunnyvale, CA, USA) was assumed to be widely available as a "triage test" under routine programmatic conditions, based on the current worldwide implementation process and scale-up. In that scenario, whether the bacilli harboured by the TB patient are rifampicin susceptible or not will be known from the start. Subsequently, profiles were developed for the treatment of rifampicin-susceptible (RS) and rifampicin-resistant (RR)-TB, respectively, the latter being considered a proxy for MDR-TB. In addition, premised on the potential for a regimen of three to four entirely new anti-TB drugs (i.e. excluding rifampicin, isoniazid and pyrazinamide) for which minimal or no resistance would exist as a result of prior use in the community, a TRP was developed for "pan-TB treatment". This regimen would be implemented in a simple and streamlined manner without need for drug-susceptibility testing (DST) or for a separate treatment pathway for patients with at least RR-TB (then, whichever rapid tests of drug resistance might be developed/available, they would suitably come as a complement to further refine the patients' needs given the resistance profile).

Mathematical modelling was then used to estimate the relative impact of selected regimen characteristics on population-wide TB incidence and mortality. The selected characteristics were 1) efficacy (achieving high nonrelapsing cure rates), 2) treatment duration, 3) adherence, 4) medical contraindications, 5) barrier to resistance and 6) baseline prevalence of resistance to drugs in the regimen. The influence of each characteristic on expected incidence and mortality outcomes was evaluated as the characteristic ranged from a minimum acceptable value to an optimistic target. 
Then, an Internet-based survey was conducted to identify what would be the priority attributes that a wide audience of stakeholders would value for the development of the TRPs. It contained core questions in four main categories (efficacy, safety, adherence and operational considerations) [26]. Finally, a web-based Delphi consultation was organised to gather input from the larger TB control and research community, and a consensus meeting took place to review the draft target profiles. The final document was formally launched at the European Respiratory Society International Congress in London (figure 1).

The details of the three TRPs are presented below.

\section{TRP for RS-TB}

Rationale

Despite the wide availability of a highly efficacious, low-cost regimen of 6 months' duration for the treatment of RS-TB, improvements are still needed if we are to achieve the WHO targets set within the context of the End TB Strategy [27]. The current 6-month regimen has several limitations including drug-related adverse events, challenging drug-drug interactions (in particular, with some antiretroviral medicines) and difficulty in ensuring adherence for the full duration of treatment across all settings. By improving adherence to treatment, shorter regimens would result in better outcomes and lower risk of acquisition of resistance, faster recovery, a shorter period at risk of side-effects, and lower patient and programme costs $[6,7]$. Future RS-TB regimens would ideally also be active against strains that are monoresistant to any other first-line drug except rifampicin.

\section{Key attributes}

The characteristics of the TRP for RS-TB treatment are summarised in table 1 . To achieve these, it will be necessary to use multiple-drug combinations with both bactericidal and sterilising efficacy according to well established principles of TB chemotherapy [1] to assure durable and relapse-free cure.

\section{TRP for RR-TB}

Rationale

About 480000 new MDR-TB cases and an additional 100000 cases of RR disease were estimated to have occurred in the world in 2015 [28]. However, only 125000 MDR-TB patients were reported by countries to have initiated treatment [28]. The conformity of these regimens to those recommended by WHO and the quality of medicines used is usually unknown, and only about one half of patients treated globally is reported to complete treatment successfully $[28,29]$.

In the absence of formal randomised controlled trials, the conventional (or longer) MDR-TB treatment recommended by WHO (based on a minimum of five drugs) is the result of expert opinion based on observational studies [30] and there has been no head-to-head comparison of one MDR regimen versus another MDR regimen of any kind (of note, a trial is currently underway to evaluate a short MDR regimen versus the 20-month WHO standard) [31, 32]. As a consequence, using the GRADE system definitions, the available evidence is generally of "low" or "very low" quality [33]. In addition, the complexity and limited efficacy of current regimens may predispose to development of additional resistance $[23,24]$. Finally, it is imperative to lower the cost of MDR-TB regimens to make them accessible in the poorest settings $[16,17]$.

FIGURE 1 Picture of the official launch of the World Health Organization document at the European Respiratory Society International Congress 2016.

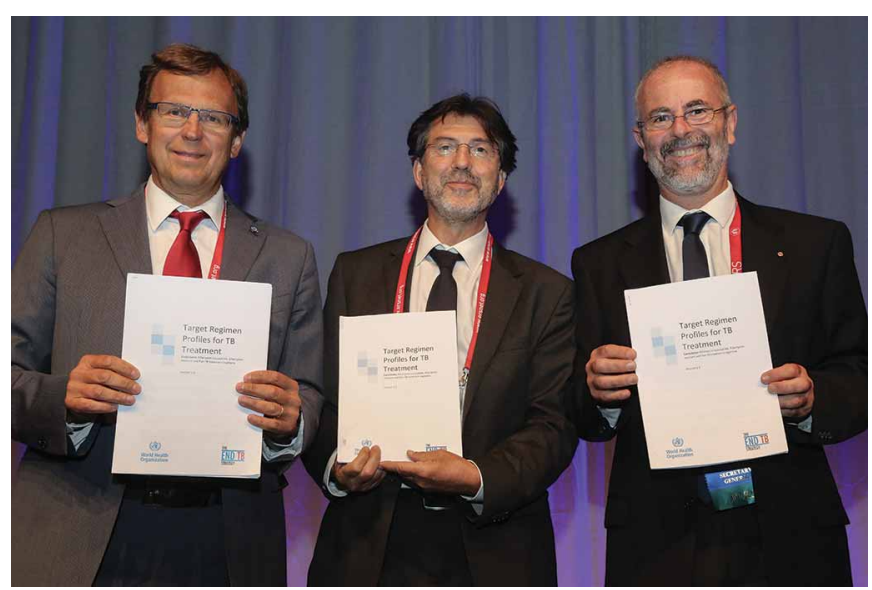


TABLE 1 Characteristics of the target regimen profile for rifampicin-susceptible (RS) tuberculosis (TB)

\section{Attribute}

1 Indication

2 Efficacy and duration of treatment

3 Target population

4 Safety and tolerability

5 DDI and metabolism

6 Formulation dosage and route of administration

7 Stability/shelf life

8 Special populations

9 Barrier to emergence of drug resistance I propensity to develop resistance, generation of cross-resistance)
Minimum ${ }^{\#}$

Active against RS M. tuberculosis strains

A regimen of 4 months or less with efficacy not inferior $^{+}$to the current standard of care 6-month regimen for DS-TB

All age groups, irrespective of HIV status

Incidence and severity of adverse events no worse than for standard of care

No more than monthly clinical monitoring and no laboratory monitoring for drug toxicity needed except in special populations (pre-existing liver disease, diabetes, etc.)

Ability to use safely without active laboratory testing or monitoring with:

First-line ART regimen(s)

Rifamycins (if a rifamycin is included in the regimen)

Drugs that induce or inhibit P450 liver enzymes

Proarrhythmic drugs that prolong QT/QTc interval

Formulation to be oral for all drugs in regimen, including paediatrics

Well tolerated and simple to administer to enhance adherence

Without cold storage requirements, with shelf lives of at least 3 years for all the drugs comprising the regimen

Safe on a wide range of patients /children, pregnant women and patients with comorbidities (HIV, viral hepatitis, diabetes, etc.) and low to no drug-drug interactions

Each component of the regimen should have no greater mutation rate (in unselected bacterial population) than 1 in $10^{7}$ mutations per bacterium per generation

New resistance to one or more drugs in the regimen emerges in $<1 \%$ of treatment courses when taken as prescribed and when no pre-existing resistance to the drugs in the regimen exists

\section{Optimum}

Active against RS M. tuberculosis strains including monoresistance to any drug except rifampicin

A regimen of 2 months or less with efficacy not inferior to the current standard of care 6-month regimen for drug-susceptible TB

All age groups, irrespective of HIV status

Incidence and severity of adverse events better than for standard of care

No active clinical monitoring and no laboratory monitoring for drug toxicity needed except in special populations (pre-existing liver disease diabetes, etc.)

No dose adjustment with other medications and ability to use safely without active laboratory testing or monitoring with:

First-line ART regimens and co-trimoxazole

Rifamycins (if a rifamycin is included in the regimen]

Drugs that induce or inhibit P450 liver enzymes

Proarrhythmic drugs that prolong QT/QTc interval

Exclusively oral delivery (preferably once daily); ideally without the need for weight band adjustments, and suitable for fixed dose combination formulations

Parenteral formulations would allow to treat severe cases

3 or fewer pills per day

Without cold storage requirements, with shelf lives of at least 5 years for all the drugs comprising the regimen

For women of child bearing potential and pregnant women, availabile human data that do not indicate that the component drugs increase the overall risk of structural abnormalities and the drugs are safe with breastfeeding

Each component of the regimen should have no greater mutation rate (in unselected bacterial population) than 1 in $10^{9}$ mutations per bacterium per generation

Essentially no acquired resistance $(<0.01 \%)$ when regimen is taken as prescribed and no pre-existing resistance to the drugs in the regimen exists

M. tuberculosis: Mycobacterium tuberculosis; ART: antiretroviral therapy; DS: drug-susceptible; DDI: drug-drug interaction. " : should be considered as a potential go/no go decision point; ${ }^{\text {9: }}$ should reflect what is needed to achieve broader, deeper, quicker global health impact; ${ }^{+}$: the term "not inferior" is intentionally used in place of noninferiority, which is a trials design and methodology term.

The newly WHO-recommended shorter MDR-TB treatment regimen of 9-12 months has shown rates of relapse-free cure topping $85 \%$ in different Asian and African countries as part of observational studies (WHO recommends the use of this regimen conditionally among selected MDR/RR-TB patients, i.e. pulmonary $\mathrm{TB}$ cases with no previous exposure or known resistance to fluoroquinolones or injectable drugs) [34-36]. This regimen is composed of seven drugs (five of which are also part of the longer regimen) known to have good bactericidal activity against MDR-TB strains. The two newly available 
agents, bedaquiline $[9,10]$ and delamanid $[8,11]$, have shown potential to improve the efficacy of MDR regimens on top of the conventional WHO-recommended regimen. Some new drugs currently in the drug development pipeline (e.g. pretomanid and sutezolid) [37] show promise for use in MDR-TB regimens together with re-purposed drugs (e.g. linezolid, clofazimine and fluoroquinolones) [3, 38]. The goal of this TRP is to identify a suitable combination regimen early in the development process that would be safe, efficacious and of short duration [39].

\section{Key attributes}

The characteristics of this TRP are summarised in table 2. The optimal regimen is expected to be efficacious and safe in all RR-TB patients, whether they have already received TB treatment or not. DST may be needed at the start of treatment to diagnose the resistance pattern to determine whether a particular regimen is indicated. Furthermore, DST will be needed for monitoring amplification of resistance in an individual patient and resistance prevalence in a population.

In the optimal case, efficacy (bacteriological cure without relapse in at least 1-year follow-up, among patients who are not lost to follow-up) should approach that of the standard WHO regimen for DS-TB. Furthermore, it is expected that, as the efficacy of drugs included in the regimens increases, the total number of drugs constituting the regimen can decrease. This should minimise the probability of drugdrug interaction/toxicity and increase the ability to coformulate the individual drugs into fixed-dose combinations. Decreased complexity of a RR-TB regimen will likely be readily accepted by national TB programmes as they will be easier to implement.

\section{TRP for pan-TB treatment}

Rationale

A highly effective, safe and well tolerated three- to four-drug fully oral regimen that could be administered to any TB patient regardless of drug resistance profile would revolutionise the treatment of TB [26]. To allow for universal adoption, the regimens should be simple to administer (ideally once daily) and have low propensity for drug-drug interactions. As there should be no or minimal prior resistance to the drugs included in the regimen, it may be used empirically without the need for DST, eliminating treatment delays.

\section{Key attributes}

The intended use case assumes this simple, novel regimen is simultaneously studied and approved for empirical use in both RS- and RR-TB patients with strains sensitive to the new drugs. This would be particularly important in areas with high prevalence of MDR-TB and low availability of DST, where patients may be treated inappropriately and continue to transmit disease for extended periods (table 3).

\section{Forecasted role of TRPs for new regimens}

The TRPs presented here describe the series of attributes that are considered essential for novel treatment of TB, such as efficacy, safety, toxicity, drug-drug interactions and potential of acquisition of drug resistance. Satisfying all of these characteristics in a single regimen, however, will be difficult to achieve in the short term, and regimen developers might have to face trade-offs: for example, increasing efficacy (cure rates) or safety versus shortening treatment duration, or making regimens simple and well tolerated versus making them more complex and robust to emergence of drug resistance.

It should be understood that, for an infectious disease such as TB with a large global burden and ongoing person-to-person transmission, the efficacy of the new regimens will depend heavily on operational factors that also affect a regimen's ability to fulfil its role (e.g. background antimicrobial resistance, resistance in the MDR-TB patient population to important existing TB drugs, development of resistance to new drugs and slow uptake of new drugs). For these reasons, these TRPs give indications on the respective attributes to be considered at the developmental stage, but these should not be dissociated from the factors to be considered at implementation stage.

All drugs used in a study regimen should meet either WHO prequalification or certification from a stringent regulatory authority or be study drugs that are tested in a facility with good manufacturing practice certification for quality assurance. It would be suitable that each individual drug component or the regimen as a whole be approved for use in $\mathrm{TB}$ by at least one stringent regulatory authority. If a regimen is recommended by the WHO using GRADE evidence review, it is expected that the regimen, or its individual components, be widely available in quality assured formulations within 2 years.

Strategies to lower the regimen costs should be considered from the outset, with adherence to the principles of access to medicine. Once a new regimen is established to be superior to current regimens in terms of safety and/or efficacy, then stakeholders should continue to work to bring down the cost of the regimen by working on costs of individual drugs, as well increasing the demand for the new regimen. Finally, it is 
TABLE 2 Characteristics of the target regimen profile for rifampicin-resistant (RR) tuberculosis (TB)

\section{Attribute}

1 Indication

2 Efficacy and duration of treatment

3 Target population in respect to age

4 Safety and tolerability

5 DDI and metabolism

6 Formulation dosage, route of administration and dosing lincluding schedule)

7 Stability/shelf-life

8 Special populations

9 Barrier to emergence of drug resistance ( propensity to develop resistance, generation of cross-resistance)
Minimum"

The RR-TB regimen is indicated for patients infected with RR strains (including MDR-TB) Indication may be contingent upon additional resistance to existing first- or second-line drugs, and supported by appropriate DST

A 6-12-month treatment regimen Efficacy (bacteriological cure without relapse in at least 1-year follow-up, among patients who are not lost to follow up) should be not inferior to the WHO recommended standard of care for MDR-TB

At least adolescent (age 12-19 years) and adults

SAEs no more than $5 \%$ and treatment discontinuation due to TEAEs no more than $2.5 \%$

QT prolongation and proarrhythmic effects of the regimen would not put the patient at a moderate or high risk of arrhythmias or sudden death

Ability to adjust dosing or perform safe monitoring for DDls with:

At least one first-line ART regimen

Drugs that induce or inhibit P450 liver enzymes

Pro-arrhythmic QT prolonging drugs

Formulation to be oral for all drugs in regimen

Ability to deliver paediatric dosing of the regimen

Twice-daily dosing and manageable food restrictions

3 years for all drugs in the regimen No cold chain requirements

Adults and women of childbearing potential Increased acceptable risk (benefits outweigh the risk in most cases) for pregnancy women, paediatrics and those with significant renal or hepatic disease

Inclusions of patients with comorbidities including:

HIV

Diabetes

Alcoholism

Viral hepatitis

New resistance to one or more drugs in the regimen emerges in $<2 \%$ of treatment courses when taken as prescribed and when no pre-existing resistance to the drugs in the regimen exists
Optimum

The RR-TB regimen is indicated for all patients infected with RR-TB strains, with usage consistent with principles of good antibiotic stewardship

Less than or equal to 6-month treatment regimen

Efficacy should be $>90 \%$

All age groups irrespective of severity of disease, pulmonary or extrapulmonary TB, or HIV status

SAEs are no more than $2 \%$ and treatment discontinuation due to TEAEs no more than $2 \%$

The regimen would have no or insignificant QT prolongation or proarrhythmic effects

No dose adjustment with other medications and ability to safely use without active laboratory tests monitoring with:

ART regimens and co-trimoxizole

Drugs that induce or inhibit P450 liver enzymes

Pro-arrhythmic QT prolonging drugs

Formulation to be oral.

FDC formulations available (desirable to have no weight adjustment for adults)

Paediatric (oral) and i.v. formulations must also be available

Once-daily or intermittent dosing (preference for once-weekly or only monthly as the intermittency

5 years for all drugs in the regimen No cold chain requirements

Adults, paediatrics, women of childbearing potential and pregnant women

Ability to use the regimen with patients with significant renal disease

Inclusions of patients with comorbidities including:

HIV

Diabetes

Alcoholism

Viral hepatitis

Opiate addiction

Essentially no acquired resistance $(<0.1 \%)$ when regimen is taken as prescribed and no pre-existing resistance to the drugs in the regimen exists

MDR: multidrug-resistant; DST: drug-susceptibility testing; WHO: World Health Organization; SAE: serious adverse event; TEAE: treatment-emergent adverse event; DDI: drug-drug interaction; ART: antiretroviral therapy; FDC: fixed-dose combination; i.v.: intravenous. \# : should be considered as a potential go/no go decision point; ": should reflect what is needed to achieve broader, deeper, quicker global health impact. 
TABLE 3 Characteristics of the target regimen profile for pan-tuberculosis (TB) treatment

\section{Attribute}

1 Indication

2 Efficacy and duration of treatment

3 Target population

4 Safety and tolerability

5 DDI and metabolism

6 Formulation, dosage and route of administration

\section{Stability/shelf life}

8 Barrier to emergence of drug resistance (propensity to develop resistance, generation of cross-resistance)
Minimum \#

Indicated as first-line treatment for pulmonary TB without need to determine rifampicin resistance

Not inferior to RS-TB standard of care in a 6-month regimen

Indicated for all cases ladults and children, including HIV co-infected)

Incidence and severity of adverse events no worse than RS-TB standard of care

Ability to adjust dosing or perform safe monitoring for DDIs with at least one ART regimen, drugs metabolised by $\mathrm{P} 450$ liver enzymes and pro-arrhythmic QT interval prolonging drugs

Oral, once lor if balanced by exceptional performance on other attributes, twicel daily, containing $\leqslant 4$ novel antibacterial compounds Suitable for FDC

Stable for $\geqslant 3$ years in climate zones 3 and 4 at $30^{\circ} \mathrm{C} / 75 \% \mathrm{RH}$

Each component of the regimen should have no greater mutation rate (in unselected bacterial population) than 1 in $10^{7}$ mutations per bacterium per generation

New resistance to $\geqslant 1$ drugs in the regimen emerges in $<2 \%$ of treatment courses when taken as prescribed and no pre-existing resistance to the drugs in the regimen exists
Optimum

Indicated as first-line treatment for pulmonary TB without need to determine rifampicin resistance

Not inferior to RS-TB standard of care in regimen of 4 months or less

Same

Incidence and severity of adverse events better than for RS-TB standard of care

No need for dose adjustment and ability to safely use without active laboratory test monitoring Specifically, not interfering with antiretrovirals, drugs metabolised by $\mathrm{P} 450$ liver enzymes and pro-arrhythmic QT interval prolonging drugs

Oral, once-daily dosing with no special weight banding
Stable for $\geqslant 5$ years in climate zones 3 and 4 at $30^{\circ} \mathrm{C} / 75 \% \mathrm{RH}$

Each component of the regimen should have no greater mutation rate (in unselected bacterial population) than 1 in $10^{9}$ mutations per bacterium per generation

Essentially no acquired resistance $(<0.1 \%)$ when regimen is taken as prescribed and no pre-existing resistance to the drugs in the regimen exists

RS: rifampicin-susceptible; DDI: drug-drug interaction; ART: antiretroviral therapy; FDC: fixed-dose combination; RH: relative humidity. \#: should

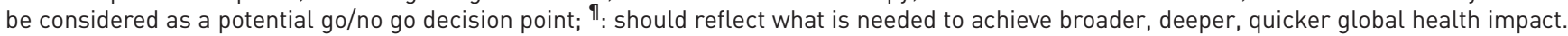

assumed that within a few years of release, the production for supply of the drugs in the new regimen could be rapidly scaled up to match demand with a corresponding decrease in the price. It is also expected that a new regimen will reduce nondrug cost aspects (e.g. monitoring visits, adherence, patient support and safety aspects), thereby improving simplicity of use, and these benefits may offset increased drug costs.

In conclusion, the TRPs proposed in this document represent a milestone towards the development of new regimens for the treatment of all forms of TB, and will serve consortia linking drug developers, academics, researchers, public health institutions and nongovernmental organisations.

\section{References}

1 Grosset J. Bacteriologic basis of short-course chemotherapy for tuberculosis. Clin Chest Med 1980; 1: 231-241.

2 Falzon D, Jaramillo E, Schunemann HJ, et al. WHO guidelines for the programmatic management of drug-resistant tuberculosis: 2011 update. Eur Respir J 2011; 38: 516-528.

3 Hughes J, Isaakidis P, Andries A, et al. Linezolid for multidrug-resistant tuberculosis in HIV-infected and -uninfected patients. Eur Respir J 2015; 46: 271-274.

4 Nahid P, Dorman SE, Alipanah N, et al. Official American Thoracic Society/Centers for Disease Control and Prevention/Infectious Diseases Society of America clinical practice guidelines: treatment of drug-susceptible tuberculosis. Clin Infect Dis 2016; 63: e147-e195.

5 Pontali E, Sotgiu G, D'Ambrosio L, et al. Bedaquiline and multidrug-resistant tuberculosis: a systematic and critical analysis of the evidence. Eur Respir J 2016; 47: 394-402.

6 Lienhardt C, Raviglione M, Spigelman M, et al. New drugs for the treatment of tuberculosis: needs, challenges, promise, and prospects for the future. J Infect Dis 2012; 205: Suppl. 2, S241-S249. 
7 Zumla A, Chakaya J, Centis R, et al. Tuberculosis treatment and management - an update on treatment regimens, trials, new drugs, and adjunct therapies. Lancet Respir Med 2015; 3: 220-234.

8 Gler MT, Skripconoka V, Sanchez-Garavito E, et al. Delamanid for multidrug-resistant pulmonary tuberculosis. N Engl J Med 2012; 366: 2151-2160.

9 Pym AS, Diacon AH, Tang SJ, et al. Bedaquiline in the treatment of multidrug- and extensively drug-resistant tuberculosis. Eur Respir J 2016; 47: 564-574.

10 World Health Organization. The use of bedaquiline in the treatment of multidrug-resistant tuberculosis: interim policy guidance (WHO/HTM/TB/2013.6). Geneva, World Health Organization, 2013.

11 World Health Organization. The use of delamanid in the treatment of multidrug-resistant tuberculosis: interim policy guidance (WHO/HTM/TB2014.23). Geneva, World Health Organization, 2014.

12 RESIST-TB. DR-TB Clinical Trials Progress Report. www.resisttb.org/?page_id=1602

13 Migliori GB, Lienhardt C, Weyer K, et al. Ensuring rational introduction and responsible use of new TB tools: outcome of an ERS multisector consultation. Eur Respir J 2014; 44: 1412-1417.

14 Nunn AJ, Rusen ID, Van Deun A, et al. Evaluation of a standardized treatment regimen of anti-tuberculosis drugs for patients with multi-drug-resistant tuberculosis (STREAM): study protocol for a randomized controlled trial. Trials 2014; 15: 353.

15 Working Group for New TB Drugs. Clinical pipeline. www.newtbdrugs.org/pipeline/clinical Date last accessed: September 19, 2016.

16 Gospodarevskaya E, Tulloch O, Bunga C, et al. Patient costs during tuberculosis treatment in Bangladesh and Tanzania: the potential of shorter regimens. Int J Tuberc Lung Dis 2014; 18: 810-817.

17 Diel R, Vandeputte J, de Vries G, et al. Costs of tuberculosis disease in the European Union: a systematic analysis and cost calculation. Eur Respir J 2014; 43: 554-565.

18 Winters N, Butler-Laporte G, Menzies D. Efficacy and safety of World Health Organization group 5 drugs for multidrug-resistant tuberculosis treatment. Eur Respir J 2015; 46: 1461-1470.

19 Semvua HH, Kibiki GS, Kisanga ER, et al. Pharmacological interactions between rifampicin and antiretroviral drugs: challenges and research priorities for resource-limited settings. Ther Drug Monit 2015; 37: 22-32.

20 Tiberi S, Payen MC, Sotgiu G, et al. Effectiveness and safety of meropenem/clavulanate-containing regimens in the treatment of MDR- and XDR-TB. Eur Respir J 2016; 47: 1235-1243.

21 Cegielski JP, Dalton T, Yagui M, et al. Extensive drug resistance acquired during treatment of multidrug-resistant tuberculosis. Clin Infect Dis 2014; 59: 1049-1063.

22 Vasakova M. Challenges of antituberculosis treatment in patients with difficult clinical conditions. Clin Respir J 2015; 9: 143-152.

23 Koser CU, Javid B, Liddell K, et al. Drug-resistance mechanisms and tuberculosis drugs. Lancet 2015; 385: 305-307.

24 Gao J, Ma Y, Du J, et al. Later emergence of acquired drug resistance and its effect on treatment outcome in patients treated with Standard Short-Course Chemotherapy for tuberculosis. BMC Pulm Med 2016; 16: 26.

25 Food and Drug Administration. Guidance for Industry and Review Staff: Target Product Profile - a Strategic Development Process Tool. www.fda.gov/downloads/drugs/guidancecomplianceregulatoryinformation/guidances/ ucm080593.pdf

26 World Health Organization. Target Regimen Profiles for TB Treatment (WHO/HTM/TB/2016.16). Geneva, World Health Organization, 2016.

27 Uplekar M, Weil D, Lonnroth K, et al. WHO's new End TB Strategy. Lancet 2015; 385: 1799-1801.

28 World Health Organization. Global tuberculosis report 2016 (WHO/HTM/TB/2016.13). Geneva, World Health Organization, 2016.

29 Falzon D, Gandhi N, Migliori GB, et al. Resistance to fluoroquinolones and second-line injectable drugs: impact on MDR-TB outcomes. Eur Respir J 2012; 42: 156-168.

30 World health Organization. Guidelines for the programmatic management of drug-resistant tuberculosis-2011 update. (WHO/HTM/TB/2011.6). Geneva, World Health Organization, 2011.

31 Ahuja SD, Ashkin D, Avendano M, et al. Multidrug resistant pulmonary tuberculosis treatment regimens and patient outcomes: an individual patient data meta-analysis of 9,153 patients. PLoS Med 2012; 9: e1001300.

32 Falzon D, Mirzayev F, Wares F, et al. Multidrug-resistant tuberculosis around the world: what progress has been made? Eur Respir J 2015; 45: 150-160.

33 WHO treatment guidelines for drug-resistant tuberculosis - 2016 update (WHO/HTM/TB/2016.04). Geneva, World Health Organization, 2016.

34 Van Deun A, Maug AKJ, Salim MAH, et al. Short, highly effective, and inexpensive standardized treatment of multidrug-resistant tuberculosis. Am J Respir Crit Care Med 2010; 182: 684-692.

35 Kuaban C, Noeske J, Rieder H, et al. High effectiveness of a 12-month regimen for MDR-TB patients in Cameroon. Int J Tuberc Lung Dis 2015; 19: 517-524.

36 Piubello A, Harouna SH, Souleymane M, et al. High cure rate with standardised short-course multidrug-resistant tuberculosis treatment in Niger: no relapses. Int J Tuberc Lung Dis 2014; 18: 1188-1194.

37 Dawson R, Diacon AH, Everitt D, et al. Efficiency and safety of the combination of moxifloxacin, pretomanid (PA-824), and pyrazinamide during the first 8 weeks of antituberculosis treatment: a phase $2 \mathrm{~b}$, open-label, partly randomised trial in patients with drug-susceptible or drug-resistant pulmonary tuberculosis. Lancet 2015; 385: 1738-1747.

38 Sotgiu G, Centis R, D'Ambrosio L, et al. Efficacy, safety and tolerability of linezolid containing regimens in treating MDR-TB and XDR-TB: systematic review and meta-analysis. Eur Respir J 2012; 40: 1430-1442.

39 Brigden G, Nyang'wa B-T, du Cros P, et al. Principles for designing future regimens for multidrug-resistant tuberculosis. Bull World Health Organ 2014; 92: 68-74. 